\title{
Chapter 28 \\ LEAP-UCD-2017 Type-B Predictions Through FLIP at Kyoto University
}

\author{
Kyohei Ueda and Toma Wada
}

\begin{abstract}
This study reports the results of type-B predictions for dynamic centrifuge model tests of a liquefiable sloping ground conducted at various centrifuge facilities within a framework of the LEAP-UCD-2017. The simulations are carried out with a finite strain analysis program, called "FLIP TULIP," which incorporates a strain space multiple mechanism model based on the finite strain theory (including both total and updated Lagrangian formulations). The program can take into account the effect of geometrical nonlinearity as well as material nonlinearity's effect. Soil parameters for the constitutive model are determined referring to the results of laboratory experiments (e.g., cyclic triaxial tests) and some empirical formulae. This chapter describes the parameter identification process in details as well as the computational conditions (e.g., geometric modeling, initial and boundary conditions, numerical schemes such as time integration technique). Type-B prediction results are compared with the centrifuge test results to examine the applicability of the program and constitutive model.
\end{abstract}

\subsection{Introduction}

To estimate liquefaction-induced damage to soil-structure systems during huge earthquakes, analytical techniques (e.g., effective stress analysis) have been actively studied as well as experimental ones (e.g., laboratory soil test, centrifuge model test) since 1970s. In particular, research on constitutive laws of sandy (or granular) materials has been dramatically developed by academic researchers since 1990s for its application in practice; an effective stress analysis technique incorporating the constitutive laws is being used more and more in seismic design to estimate the

\footnotetext{
K. Ueda $(\bowtie)$

Disaster Prevention Research Institute, Kyoto University, Uji, Kyoto, Japan

e-mail: ueda.kyohei.2v@kyoto-u.ac.jp

T. Wada

Department of Civil and Earth Resources Engineering, Kyoto University, Kyoto, Japan

e-mail: wada.touma.67a@st.kyoto-u.ac.jp 
damage level of soil-structure systems due to liquefaction. Laboratory and model experimental results are often used for validation of the analysis technique, and such constitutive models have been modified and updated if need arises. However, a practical way for the validation process (e.g., how to assess the validity of constitutive models) has not yet been established, in particular for dynamic liquefactioninduced behavior of soil-structure systems, as well known in geotechnical engineer/ researcher community.

The importance of validation was already pointed out in the VELACS project more than 20 years ago (Arulanandan and Scott 1993/1994). The project was an opportunity for geotechnical researchers to realize the need for improvement of numerical modeling on liquefiable ground. In addition, the project has revealed that reliable data for the validation process were difficult to obtain from laboratory and/or centrifuge experimental results because such experimental results generally have some variation among different facilities, particularly in the case of experiments on complicated phenomena such as liquefaction. To answer the problems that were pointed out but could not be solved in the VELACS project, some researchers have proposed a new international collaborative effort called "LEAP" (Liquefaction Experiment and Analysis Projects) (Iai 2015; Kutter et al. 2015; Manzari et al. 2015; Zeghal et al. 2015). One of the main goals is to establish a practical way for validating the capabilities of existing analytical techniques for liquefaction-induced behavior, including constitutive laws of granular materials, through comparison with laboratory and centrifuge experiments.

As part of LEAP exercises, LEAP-GWU-2015 aimed to obtain a set of highquality experimental data among different centrifuge facilities; a sloping liquefiable ground was built up in a rigid model container, having a simpler lateral boundary condition rather than a laminar container, in order to avoid difficulties in numerical modeling associated with complex boundary conditions in the latter case. Model specifications in LEAP-GWU-2015 are presented by Kutter et al. (2018), in which the results of centrifuge experiments performed at Cambridge University (CU) in the UK, Kyoto University (KU) in Japan, National Central University (NCU) in Taiwan, Rensselaer Polytechnic Institute (RPI) and University of California Davis (UCD) in the USA, and Zhejiang University (ZU) in China are compared. More recently, LEAP-UCD-2017 has started to obtain a sufficient number of centrifuge experimental results for a sloping liquefiable ground with higher accuracy and to quantify the magnitude of variability among different centrifuge facilities.

This chapter presents results of numerical simulations for the dynamic centrifuge model tests, within a framework of type-B prediction (e.g., Lambe (1973)) phases of the LEAP-UCD-2017. The simulations are carried out with a finite strain, effective stress finite element (FE) program, called "FLIP TULIP," which incorporates a strain space multiple mechanism model based on the finite strain theory (including both total and updated Lagrangian formulations) (Ueda 2009; Iai et al. 2013). The program can take into account the effect of geometrical nonlinearity as well as that of material nonlinearity. This chapter describes the identification procedure of input model parameters in details as well as the computational conditions such as geometrical modeling, initial, and boundary conditions. 


\subsection{Brief Overview of Centrifuge Experiments}

Vargas et al. (2019) describe the centrifuge modeling equipment used at Disaster Prevention Research Institute, Kyoto University, and Kutter et al. (2019a, b) present the detailed specifications for the LEAP-UCD-2017 experiments conducted at all of the centrifuge facilities. This section briefly describes a summary of the centrifuge model tests. A sketch of the model is shown in Fig. 28.1. The experiments modeled submerged Ottawa F65 sand (a target relative density of about 65\%) with a 5-degree sloping ground surface in a rigid box subjected to ramped sinusoidal wave motions ( $1 \mathrm{~Hz}, 16$ cycles). At some facilities (e.g., Kyoto) in which the direction of horizontal shaking is in the plane of spinning of the centrifuge, the slope in the shaking direction was built up as a curved surface considering the effective radius from the axis of rotation of the centrifuge (Fig. 28.1b). The sloping ground had the width of

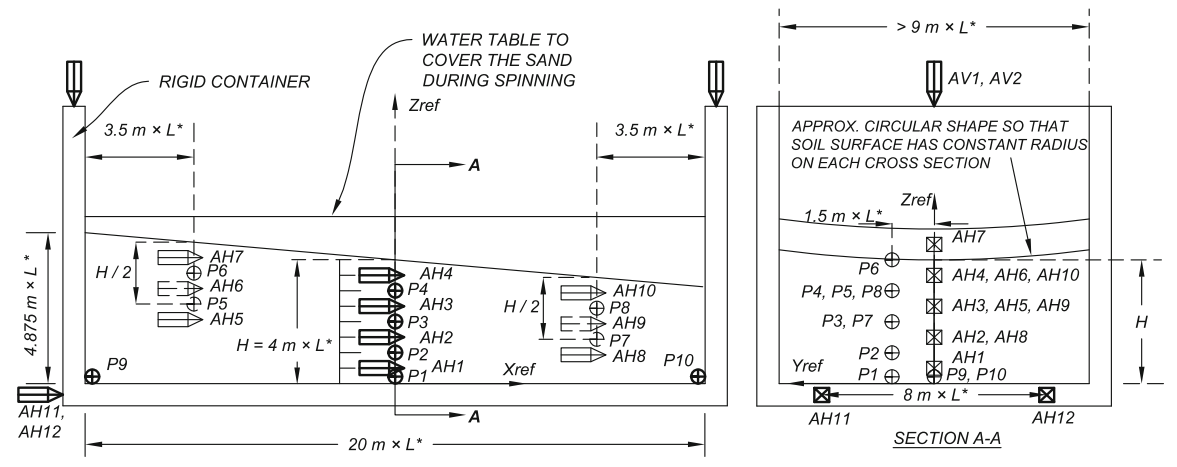

(a)

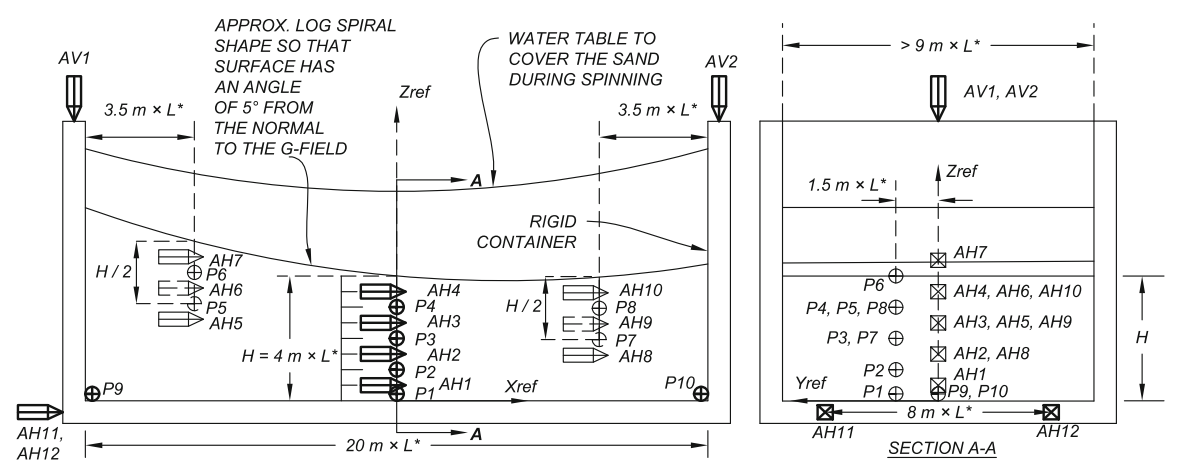

(b)

Fig. 28.1 Schematic for LEAP-UCD-2017 centrifuge model tests (Kutter et al. 2019a): (a) Sectional drawing for shaking parallel to the axis of the centrifuge (ZU, RPI, NCU). (b) Sectional drawing for shaking in the plane of spinning of the centrifuge (UCD, Kyoto, Cambridge) 
$20 \mathrm{~m}$ and the height of $4 \mathrm{~m}$ at midpoint in prototype scale. The input motions used for LEAP-UCD-2017 validation experiments consisted of three nondestructive motions (i.e., motions 1, 3, and 5) and two destructive motions (i.e., motions 2 and 4): the first destructive motion, which corresponded to the second motion of the sequence (motion 2), had an acceleration amplitude of $0.15 \mathrm{~g}$. The nondestructive motions were applied to evaluate the ground properties (e.g., shear wave velocity) after each destructive motion. In this chapter, we focus on the dynamic responses under the first destructive motion (motion 2) for validation of the effective stress FE program (i.e., FLIP TULIP) for liquefaction problems incorporating the strain space multiple mechanism model.

\subsection{Constitutive Model of Soils}

As is the case with the type-B and -C simulations for LEAP-GWU-2015 (Ueda and Iai 2018), a strain space multiple mechanism model based on the finite strain theory (Ueda 2009; Iai et al. 2013) is used for LEAP-UCD-2017 type-B predictions. The original version of the model was proposed within the context of the infinitesimal strain theory (Iai et al. 1992), and implemented in a FE program called "FLIP ROSE" (Finite Element Analysis Program of LIquefaction Process/Response Of Soil-structure Systems during Earthquakes). The program has been frequently used in design practice for evaluating the seismic performance of soil-structure systems, particularly port structures, in Japan (Iai et al. 1992; Iai et al. 1995; Ozutsumi et al. 2002). On the basis of a multitude of virtual simple shear mechanisms oriented in an arbitrary direction, the model has an ability to simulate the evolution of induced fabric under various complicated loading conditions (e.g., the rotation of principal stress axis direction). About 10 years ago, a new stress-dilatancy relationship was introduced in the constitutive model for controlling dilative (or positive) and contractive (or negative) components of dilatancy in a more sophisticated manner (Iai et al. 2011).

With an aim to take into account the effect of geometrical nonlinearity in addition to material nonlinearity's effect, the model was extended within the context of the finite strain (or large deformation) theory (Ueda 2009; Iai et al. 2013). Incorporating the extended model, a finite strain FE program called "FLIP TULIP" (Finite Element Analysis Program of LIquefaction Process/Total and Updated Lagrangian Program of LIquefaction Process) has been developed based on the original infinitesimal strain program (i.e., FLIP ROSE). As might be surmised from the abbreviation, two different methods - the total Lagrangian (TL) and updated Lagrangian (UL) approaches - are available in the program. The TL approach is based on the reference (or undeformed) configuration corresponding to a fixed reference time, in which the Lagrangian (or material) description is used. On the other hand, the Eulerian (or spatial) description based on the current (or deformed) configuration is used in the UL approach. However, both approaches are theoretically equivalent to each other: the only difference is a standing position (i.e., the reference or current 
configuration). One of the major advantages in conducting both the TL and UL analyses is to be able to directly compare the numerical results obtained from different numerical schemes for validating the reliability of each numerical approach. The finite strain program begins to be used in research and design practice for evaluating the seismic performance of a soil-structure system considering its large deformation behavior (Ueda et al. 2011; Ueda et al. 2015; Ueda and Iai 2018). In this chapter, only the TL approach is applied because the UL analysis has been found to give an almost identical simulation result with the TL analyses.

\subsection{Numerical Simulation (FE Analysis)}

\subsubsection{Definition of Type-A, -B, and -C Predictions}

In this chapter, type-B predictions are carried out using the strain space multiple mechanism model extended within the context of the finite strain theory for validation of the model capability. Before proceeding to explain the analytical condition in detail, the definition of type-A, -B, and -C predictions is briefly given following Lambe (1973) as follows:

Type-A prediction: Type-A prediction is done toward a planned experiment, not targeted at an actual experiment. This means type-A is a true prediction of an event made prior to the event.

Type- $B$ prediction: Type-B prediction is targeted at an actual experiment after the experiment is conducted. The predictors can get information on as-built properties and measured input data, but no knowledge of the results is available for them.

Type-C prediction: Type-C prediction is performed after the experiment is completed, with results known to the predictors. They are able to iteratively adjust the model parameters, if necessary, to enhance the quality of their simulation results compared with observations.

\subsubsection{Model Parameters}

Most of the input parameters of the strain space multiple mechanism model were determined in the same manner as in the type-B simulations for LEAP-GWU-2015 (Ueda and Iai 2018). This section briefly describes how to set the required model parameters for liquefaction analyses.

The parameters for defining the characteristics of volumetric and shear deformation are shown in Table 28.1. In the type-B prediction, the measured density at each facility should be used. However, a significant difference in the density was not 
Table 28.1 Model parameters for deformation characteristics

\begin{tabular}{l|l|l|l}
\hline Symbol & Mechanism & Parameter designation & \\
\hline$\rho_{\mathrm{t}}$ & - & Mass density & $2.041 \mathrm{t} / \mathrm{m}^{3}$ \\
\hline$p_{\mathrm{a}}$ & - & Reference confining pressure & $100.0 \mathrm{kPa}$ \\
\hline$K_{\mathrm{L} / \mathrm{Ua}}$ & Volumetric & Bulk modulus & $2.86 \times 10^{5} \mathrm{kPa}$ \\
\hline$r_{\mathrm{K}}$ & Volumetric & $\begin{array}{l}\text { Reduction factor of bulk modulus for liquefaction } \\
\text { analysis }\end{array}$ & 0.5 \\
\hline$l_{\mathrm{K}}$ & Volumetric & Power index of bulk modulus for liquefaction analysis & 2.0 \\
\hline$G_{\mathrm{ma}}$ & Shear & Shear modulus & $1.10 \times 10^{5} \mathrm{kPa}$ \\
\hline$\phi_{\mathrm{f}}^{\mathrm{PS}}$ & Shear & Internal friction angle for plane strain & $40.0^{\circ}$ \\
\hline$h_{\max }$ & Shear & Upper bound for hysteretic damping factor & 0.24 \\
\hline
\end{tabular}

found among different facilities, and thus the value shown in Table 28.1 was used for all facilities.

The initial (small-strain) shear modulus $G_{\mathrm{m}}$ under an arbitrary confining pressure $p$ is automatically calculated in the program following the equation below:

$$
G_{\mathrm{m}}=G_{\mathrm{ma}}\left(p / p_{\mathrm{a}}\right)^{m_{\mathrm{G}}}
$$

by specifying the reference effective confining pressure $p_{\mathrm{a}}$ and the index $m_{\mathrm{G}}$, which was set to be 0.5 in this study. The index controls the dependency of shear modulus on confining pressure. The initial shear modulus $G_{\mathrm{ma}}$ under the confining pressure $p_{\mathrm{a}}$ was determined based upon the void ratio by applying an empirical relation (Ishihara 1996) as follows:

$$
G_{\mathrm{ma}}=7000 \frac{(2.17-e)^{2}}{1+e} p_{\mathrm{a}}^{0.5}
$$

As is the case with the density, the shear modulus in Table 28.1 was applied to all facilities because the difference in the shear modulus estimated from the void ratio was very small among the different facilities.

The internal friction angle $\phi_{\mathrm{f}}^{\mathrm{PS}}$ for plane strain shown in Table 28.1 was determined as follows:

$$
\sin \phi_{\mathrm{f}}^{\mathrm{PS}}=\frac{1}{2} \frac{1}{\cos (\pi / 6)} \mathrm{M}
$$

in which the critical state frictional constant $\mathrm{M}$ was estimated from a monotonic triaxial compression test under undrained condition of Ottawa F-65 sand. As shown in Table 28.1, the maximum damping constant $h_{\max }$ was set to be the standard value for sands $(=0.24)$.

In analogy with Eq. (28.1) for the shear modulus, the initial bulk modulus $K_{\mathrm{L} / \mathrm{U}}$ under an arbitrary confining pressure $p$ is automatically evaluated in the program as follows: 


$$
K_{\mathrm{L} / \mathrm{U}}=K_{\mathrm{L} / \mathrm{Ua}}\left(p / p_{\mathrm{a}}\right)^{n_{\mathrm{K}}}
$$

where $K_{\mathrm{L} / \mathrm{Ua}}$ was estimated from the shear modulus $G_{\mathrm{ma}}$ in Eq. (28.1) with the Poisson ratio of 0.33 , and the index $n_{\mathrm{K}}$, which controls the dependency of bulk modulus on confining pressure, was set to be 0.5 in this study. In the case of liquefaction analyses, the above equation is extended as

$$
K_{\mathrm{L} / \mathrm{U}}=r_{\mathrm{K}} K_{\mathrm{U} 0}\left(p / p_{0}\right)^{l_{\mathrm{K}}}
$$

where $p_{0}$ denotes the initial confining pressure, $r_{\mathrm{K}}$ is a reduction factor of bulk modulus, and the power index $l_{\mathrm{K}}$ represents the confining pressure dependency of bulk modulus (Iai et al. 2011). By changing the parameters $r_{\mathrm{K}}$ and $r_{\varepsilon_{\mathrm{d}}}$, the latter of which is a parameter for controlling both dilative and contractive components, with keeping the product (i.e., $r_{\varepsilon_{\mathrm{d}}} \times r_{\mathrm{K}}$ ) constant, volumetric characteristics due to the dissipation of excess pore water pressure (EPWP) following liquefaction can be independently controlled without altering liquefaction resistance. In this study, the standard value (i.e., 0.5) shown in Table 28.1 was used for $r_{\mathrm{K}}$ due to the lack of knowledge about the volumetric characteristics. As suggested by Ishihara and Yoshimine (1992), the characteristics can be given as a relationship between the volumetric strain due to consolidation following liquefaction and the maximum amplitude of shear strain during shaking by conducting laboratory experiments such as triaxial or torsional tests.

In addition to the centrifuge experiments, a series of laboratory tests (e.g., stresscontrolled cyclic undrained triaxial tests) were carried out by a research group from the George Washington University (El Ghoraiby et al. 2017, 2019). Figure 28.2

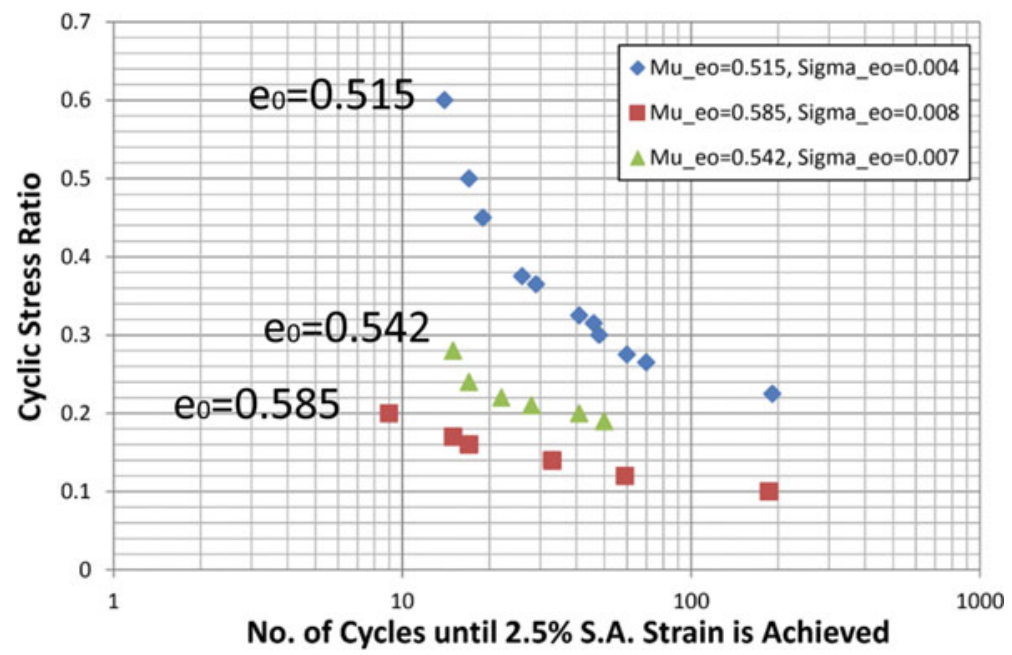

Fig. 28.2 Liquefaction strength curves from stress-controlled cyclic undrained triaxial tests (El Ghoraiby et al. 2017, 2019) 
Table 28.2 Model parameters for dilatancy

\begin{tabular}{l|l|l}
\hline Symbol & Parameter designation & \\
\hline$\phi_{\mathrm{p}}$ & Phase transformation angle & $28.0^{\circ}$ \\
\hline$\varepsilon_{\mathrm{d}}^{\mathrm{cm}}$ & Limit of contractive component & 0.15 \\
\hline$r_{\varepsilon_{\mathrm{d}}^{\mathrm{c}}}$ & Parameter controlling contractive component & 0.72 \\
\hline$r_{\varepsilon_{\mathrm{d}}}$ & Parameter controlling dilative and contractive components & 0.85 \\
\hline$q_{1}$ & Parameter controlling initial phase of contractive component & 1.0 \\
\hline$q_{2}$ & Parameter controlling final phase of contractive component & 0.1 \\
\hline$S_{1}$ & Small positive number to avoid zero confining pressure & 0.005 \\
\hline$c_{1}$ & Parameter controlling elastic range for contractive component & 1.39 \\
\hline$q_{\mathrm{us}}$ & Undrained shear strength (for steady-state analysis) & - \\
\hline
\end{tabular}

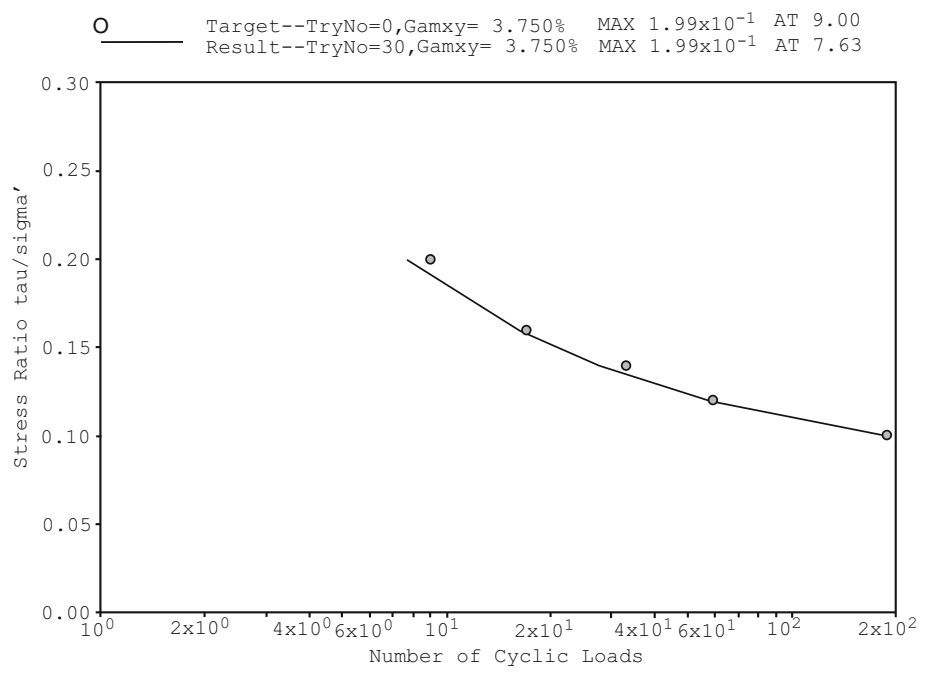

Fig. 28.3 Comparison of simulated liquefaction strength with experimental results

shows liquefaction strength curves for different three void ratios obtained from the cyclic triaxial tests. Model parameters controlling the characteristics of liquefaction and dilatancy in Table 28.2 were determined by referring to the liquefaction resistance curve for the loosest sample (i.e., $e_{0}=0.585$ ) as shown in Fig. 28.3. The simulated liquefaction strength curve was able to well capture the measured relationship between the shear stress ratio and the number of cyclic loads, including at lower stress levels. The undrained shear strength $q_{\text {us }}$ for steady-state analysis was not specified, which means $q_{\text {us }}$ was set to be an infinite value, because the strength is normally very large for clean sands such as Ottawa F-65 sand. The permeability of the ground was set to a constant value of $1.26 \times 10^{-4} \mathrm{~m} / \mathrm{s}$ to take into account the effect of pore water flow and migration; the value was determined based on permeability tests conducted at the George Washington University. 


\subsubsection{Initial/Boundary Conditions and Input Motions}

The type-B FE simulations were performed using a 2-dimensional FE mesh shown in Fig. 28.4, which has the same prototype dimension as the centrifuge experiments. The number of nodes and elements (including pore water elements) is 861 and 1600, respectively. In order to avoid shear locking and hourglass modes, the selective reduced integration (SRI) techniques (Hughes 1980) were applied to four-node quadrilateral soil elements. The FE mesh size was determined by considering the wavelength corresponding to the highest frequency of interest as suggested by Alford et al. (1974); in this study, $15 \mathrm{~Hz}$ was used for the highest frequency because the value is large enough compared to the natural frequency (i.e., $1 \mathrm{~Hz}$ ) of the input motion. For simulating the boundary conditions of the rigid box in the centrifuge experiments, displacement boundary conditions for the type-B predictions were determined as follows: the degree of freedom at the side boundaries was fixed only horizontally (i.e., vertical roller), and both horizontal and vertical displacements were fixed at the base. When it comes to the degree of freedom of pore water pressure, a hydrostatic condition was specified at the ground surface whereas the side and bottom boundaries were set to be impermeable.

Before proceeding to a seismic response (or dynamic) analysis, a self-weight analysis was carried out by applying the gravity acceleration to the model for obtaining the initial stress and strain distributions before shaking. In the seismic response analysis, the first destructive motions (motion 2) recorded at the bottom of the container during centrifuge experiments were used as an input motion for the type-B predictions; the motions were intended to be identical among all facilities, but somewhat different from the target motion depending on facilities. The numerical time integration was carried out by the SSpj method (Zienkiewicz et al. 2000) with the standard parameters $\theta_{1}=0.6$ and $\theta_{2}=0.605$ for the equation of motion and $\theta_{1}=0.6$ for the mass balance equation of pore water flow, using a time step of $0.005 \mathrm{~s}$; both equations are written in the context of the finite strain formulation

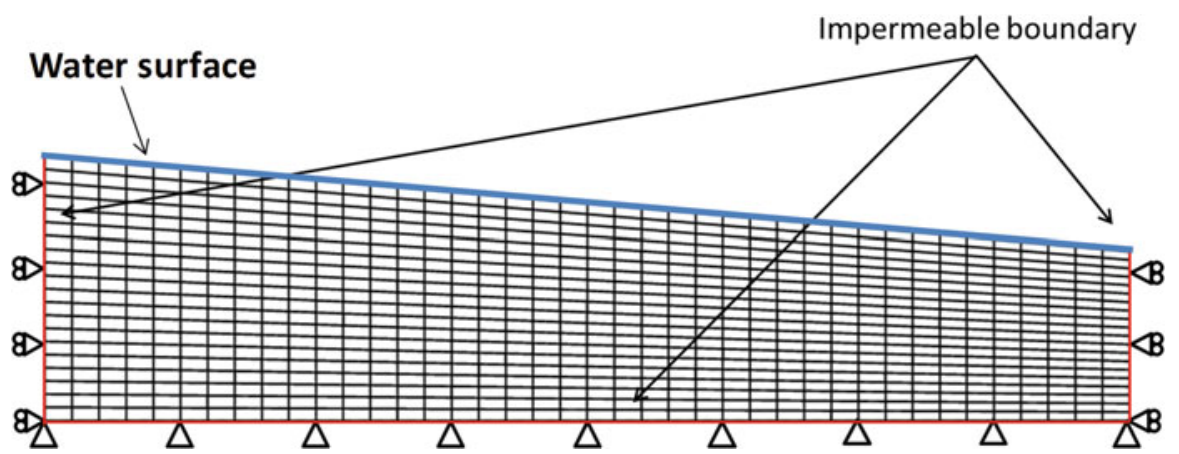

Fig. 28.4 Finite element mesh for numerical analysis 
(i.e., the Lagrangian or Eulerian description) (Ueda 2009). In the dynamic simulation, Rayleigh damping ( $\alpha=0.0, \beta=0.0002$ ) was applied for stabilizing the numerical solution process.

\subsection{Type-B Predictions (for Motion 2)}

The results of the type-B predictions are compared to centrifuge experiments in Figs. 28.5, 28.6, 28.7, 28.8, 28.9, 28.10, 28.11, 28.12, and 28.13. In each figure, simulation results, in which model parameters for dilatancy were determined referring to the liquefaction strength for $e_{0}=0.542$, are also shown. The measured responses for KyU3 in Fig. 28.5 are generally well simulated using appropriate model parameters (i.e., parameters for the closest void ratio, $e_{0}=0.585$ ) rather than the case with the parameters for $e_{0}=0.542$ : the simulated lateral displacement becomes closer to the measurement with the increase in the void ratio. The negative spikes in the measured acceleration are reasonably simulated in the type-B prediction $\left(e_{0}=0.585\right)$. However, the dynamic amplitude of simulated EPWP is overestimated to some extent. Thus, type-C simulations should be required to capture the measured behavior with higher precision.

Following is a summary of the comparison between the type-B predictions and centrifuge model tests. By setting the model parameters referring to liquefaction

\section{Experiment}
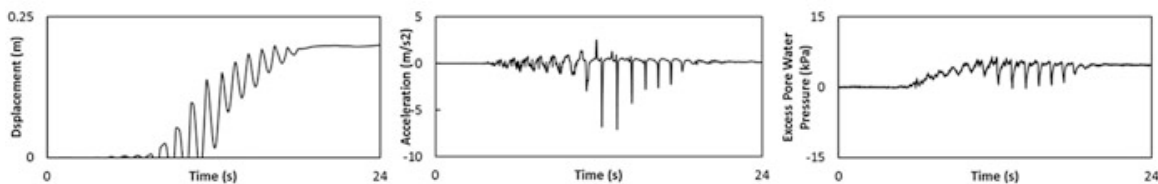

Type-B $\left(\mathrm{e}_{0}=0.585\right)$
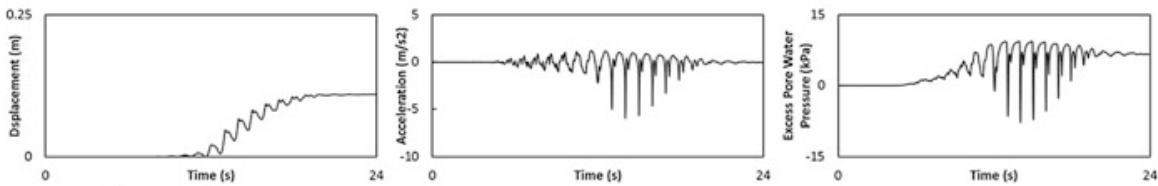

Type-B $\left(\mathrm{e}_{0}=0.542\right)$
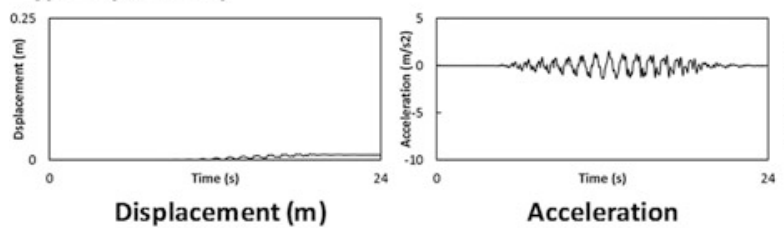

Acceleration

at $\mathrm{AH} 4(\mathrm{~m} / \mathrm{s} 2)$

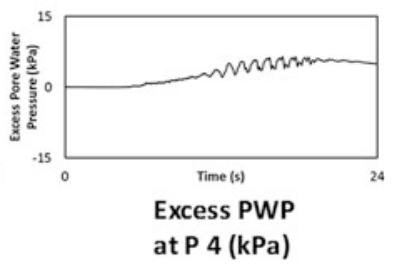

Fig. 28.5 Computed time histories (type-B prediction for KyU3, $e_{0}=0.619$ ) with experimental results 


\section{Experiment}
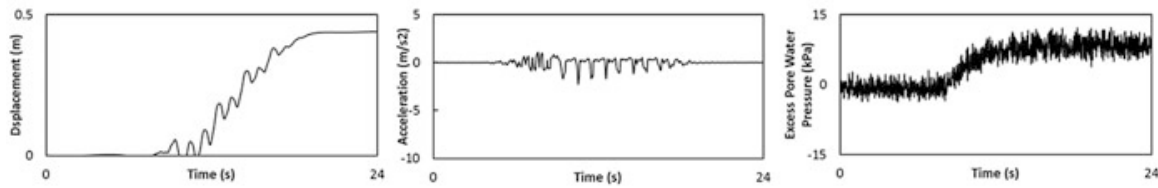

Type-B $\left(\mathrm{e}_{0}=0.585\right)$
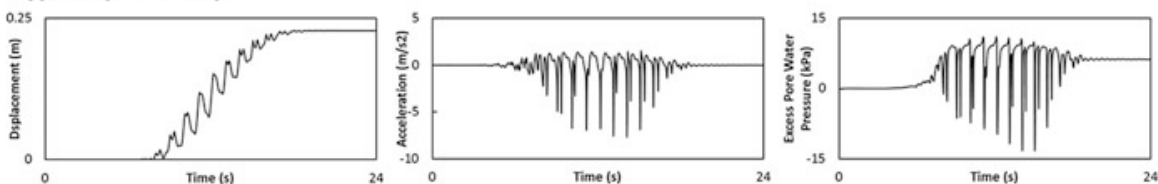

Type-B $\left(\mathrm{e}_{0}=0.542\right)$
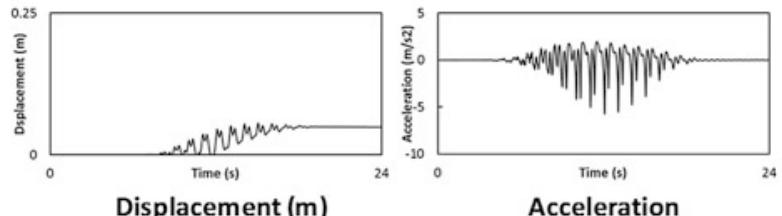

Acceleration at $\mathrm{AH} 4(\mathrm{~m} / \mathrm{s} 2)$

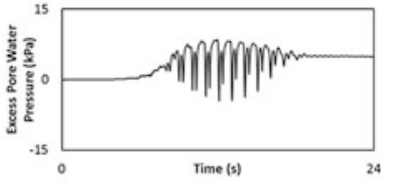

Excess PWP at $P 4(\mathrm{kPa})$

Fig. 28.6 Computed time histories (type-B prediction for $\mathrm{CU} 2, e_{0}=0.650$ ) with experimental results

\section{Experiment}

NAN
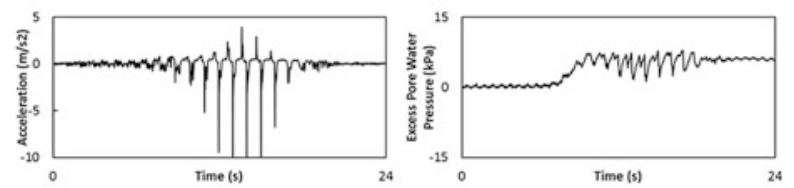

Type- $B\left(e_{0}=0.585\right)$
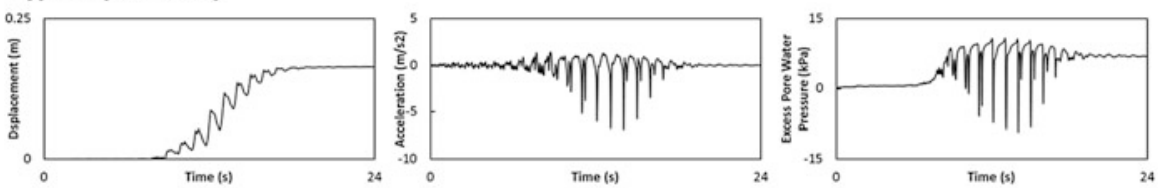

Type- $\mathrm{B}\left(\mathrm{e}_{0}=0.542\right)$
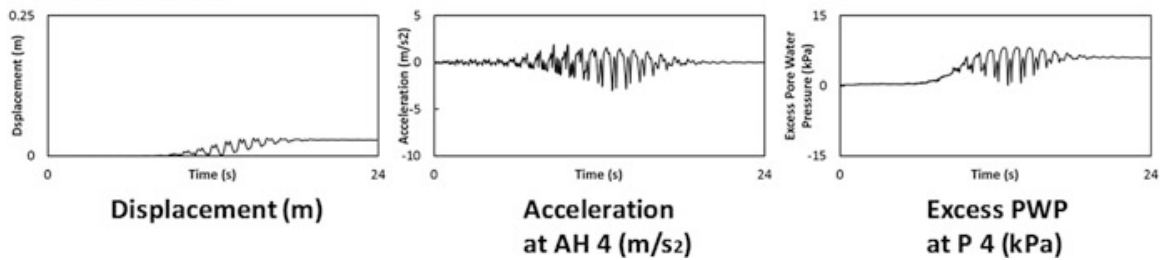

Fig. 28.7 Computed time histories (type-B prediction for KAIST1, $e_{0}=0.558$ ) with experimental results 


\section{Experiment}
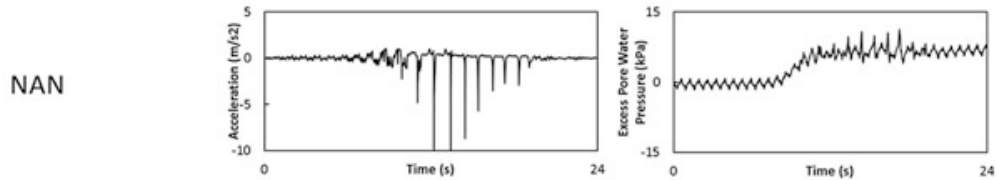

Type-B $\left(\mathrm{e}_{0}=0.585\right)$
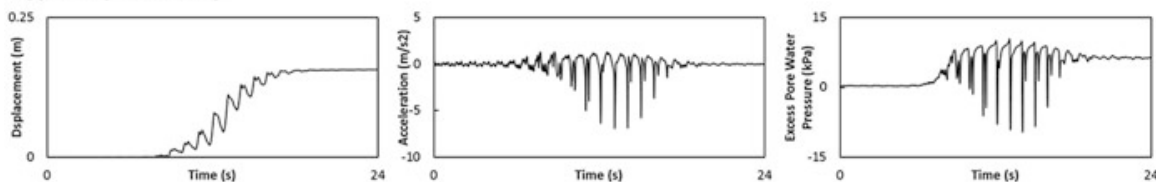

Type-B $\left(\mathrm{e}_{0}=0.542\right)$
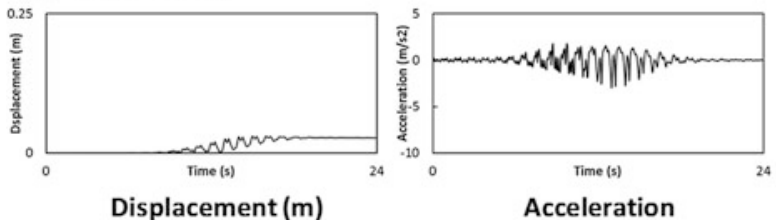

Acceleration

at $\mathrm{AH} 4\left(\mathrm{~m} / \mathrm{s}_{2}\right)$

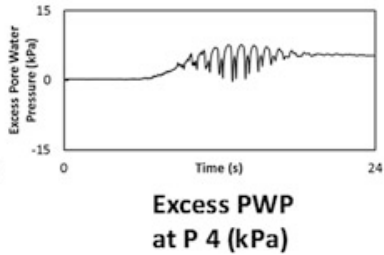

Fig. 28.8 Computed time histories (type-B prediction for KAIST2, $e_{0}=0.664$ ) with experimental results

\section{Experiment}
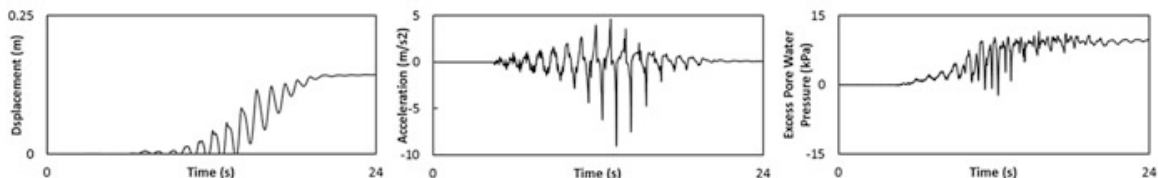

Type-B $\left(\mathrm{e}_{0}=0.585\right)$
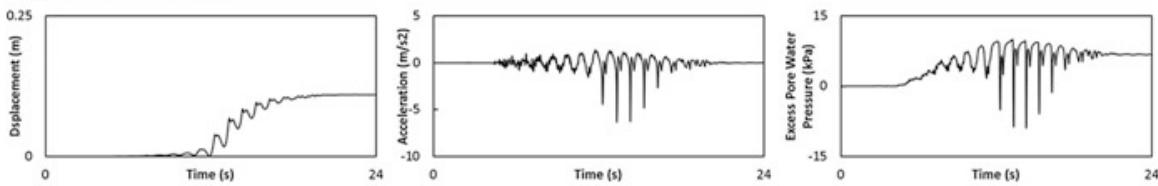

Type-B $\left(\mathrm{e}_{0}=0.542\right)$
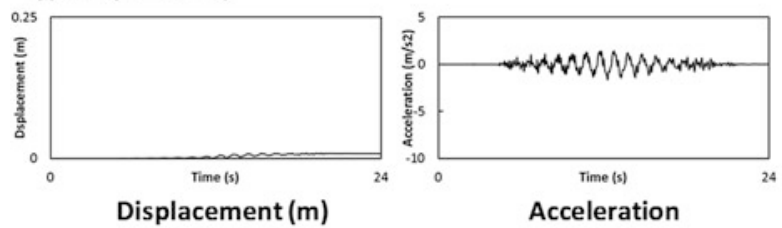

Acceleration

at $\mathrm{AH} 4(\mathrm{~m} / \mathrm{s} 2)$

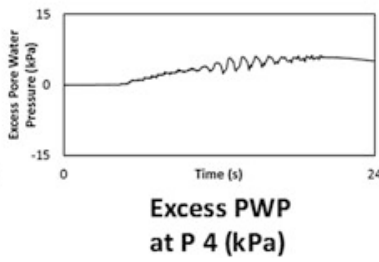

Fig. 28.9 Computed time histories (type-B prediction for UCD1, $e_{0}=0.592$ ) with experimental results 


\section{Experiment}
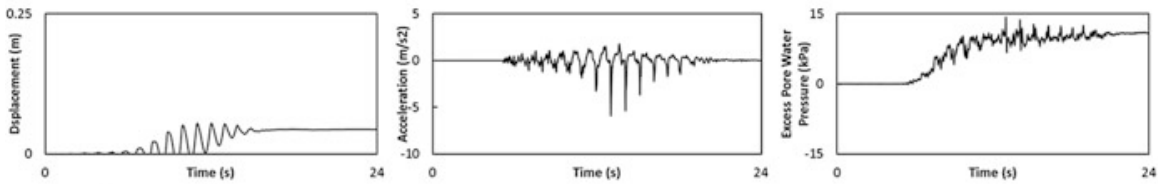

Type-B $\left(\mathrm{e}_{0}=0.585\right)$
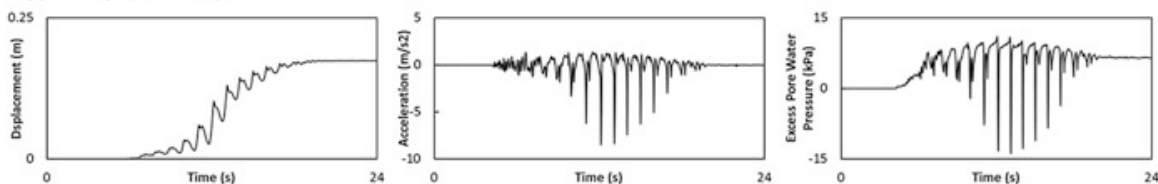

Type-B $\left(\mathrm{e}_{0}=0.542\right)$
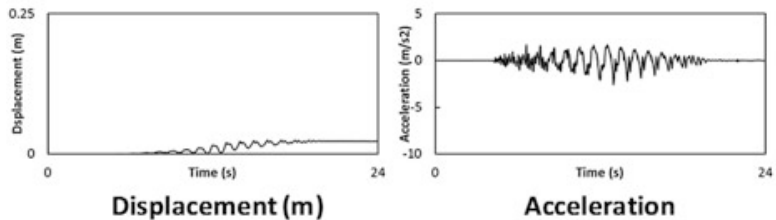

Acceleration at $\mathrm{AH} 4(\mathrm{~m} / \mathrm{s} 2)$

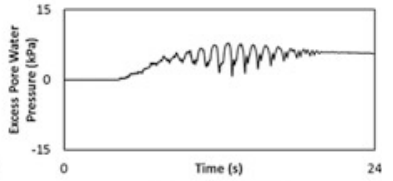

Excess PWP at P $4(\mathrm{kPa})$

Fig. 28.10 Computed time histories (type-B prediction for UCD3, $e_{0}=0.598$ ) with experimental results

\section{Experiment}
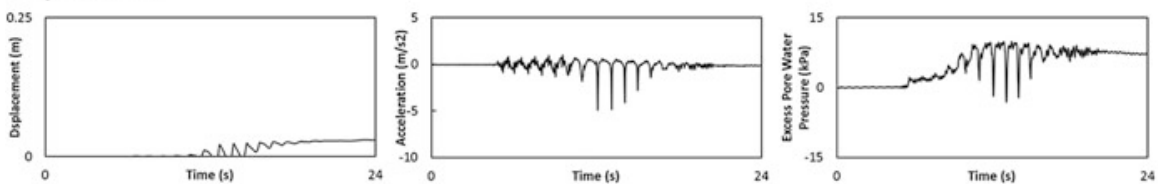

Type- $\mathrm{B}\left(\mathrm{e}_{0}=0.585\right)$
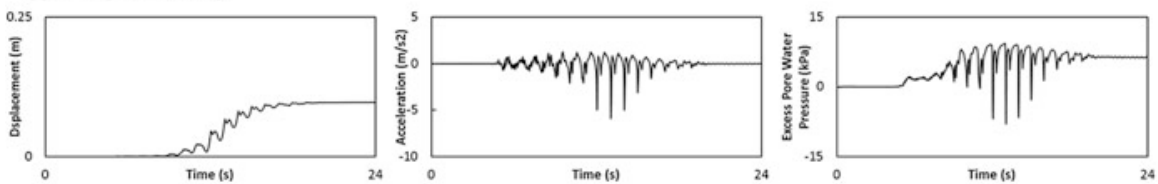

Type- $B\left(e_{0}=0.542\right)$
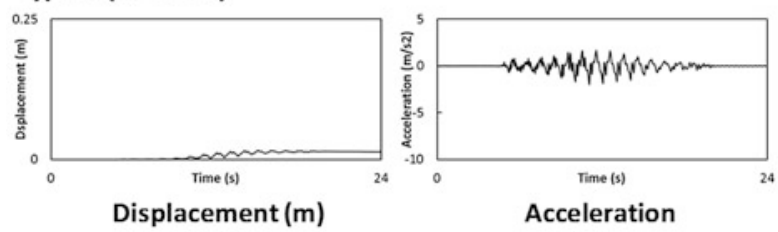

Acceleration

at AH $4(\mathrm{~m} / \mathrm{s} 2)$

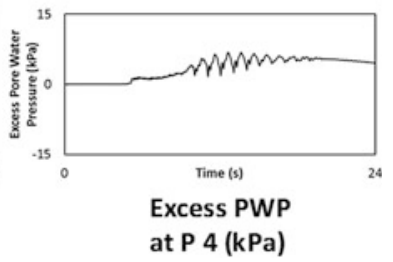

Fig. 28.11 Computed time histories (type-B prediction for ZJU2, $e_{0}=0.650$ ) with experimental results 


\section{Experiment}
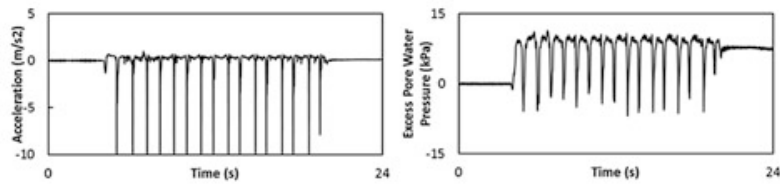

Type-B $\left(\mathrm{e}_{0}=0.585\right)$
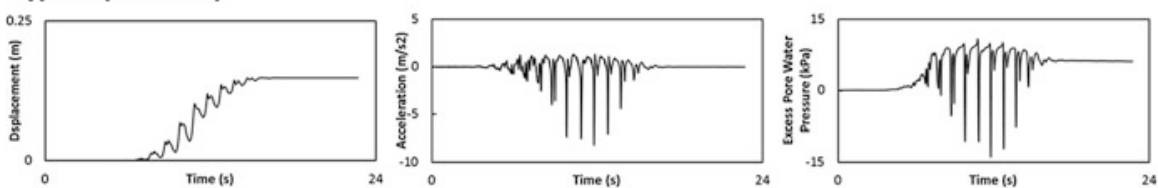

Type-B $\left(\mathrm{e}_{0}=0.542\right)$
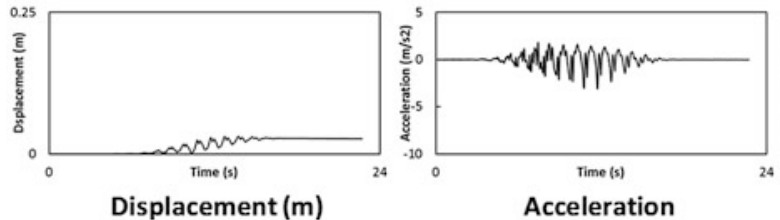

Acceleration

at $\mathrm{AH} 4(\mathrm{~m} / \mathrm{s} 2)$

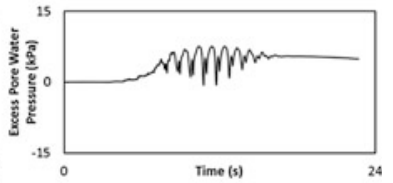

Excess PWP

at P 4 (kPa)

Fig. 28.12 Computed time histories (type-B prediction for NCU3, $e_{0}=0.604$ ) with experimental results

\section{Experiment}
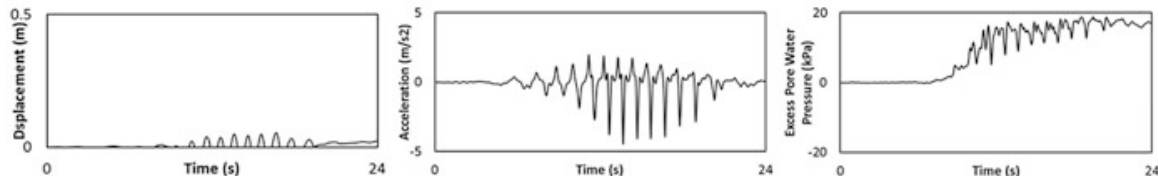

Type-B $\left(\mathrm{e}_{0}=0.585\right)$
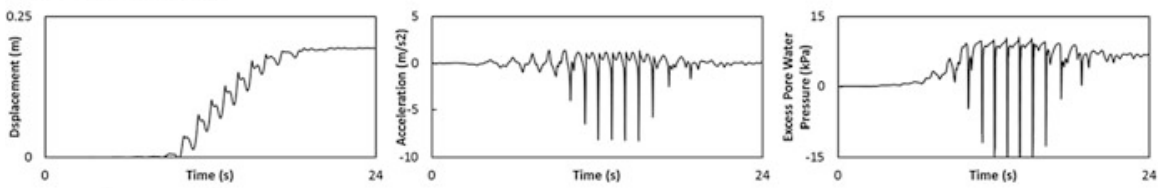

Type-B $\left(\mathrm{e}_{0}=0.542\right)$
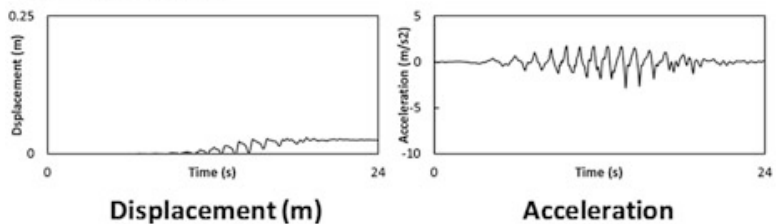

Acceleration

at $\mathrm{AH} 4(\mathrm{~m} / \mathrm{s} 2)$

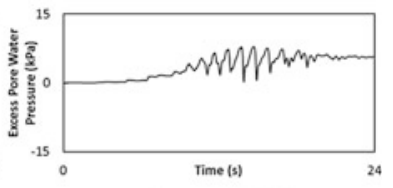

Excess PWP

at $\mathrm{P} 4(\mathrm{kPa})$

Fig. 28.13 Computed time histories (type-B prediction for Ehime2, $e_{0}=0.560$ ) with experimental results 
strength curves (Figs. 28.2 and 28.3), the prediction is able to reasonably simulate the measured lateral displacement, horizontal acceleration, and EPWP. However, it seems difficult to achieve a perfect agreement between predictions and experiments without knowledge of experimental results, even though input motions recorded during experiments can be used for the prediction. The series of centrifuge model tests conducted for LEAP-UCD-2017 show that the degree of variation in measured dynamic responses, particularly lateral displacements, among the facilities was larger than that in the input motions. This suggests that non-negligible differences in the test procedure among the facilities (e.g., how to build up the sloping ground) should be taken into account in addition to the input motion difference, if the predictor wants to simulate the experimental results more realistically.

\subsection{Conclusions}

This chapter reported the results of Type-B predictions for dynamic centrifuge model tests of a liquefiable sloping ground conducted at various centrifuge facilities within a framework of LEAP-UCD-2017. The simulations were performed with a finite strain analysis program, called "FLIP TULIP," which incorporates a strain space multiple mechanism model based on the finite strain theory (including both total and updated Lagrangian formulations). The program can consider the effect of geometrical nonlinearity as well as material nonlinearity's effect. By setting soil parameters for the constitutive model referring to the results of laboratory experiments (e.g., cyclic triaxial tests), the prediction was able to reasonably simulate the measured lateral displacement, horizontal acceleration, and excess pore water pressure. However, it was found that a perfect agreement between predictions and experiments seems difficult to achieve without knowledge of experimental results, even though input motions recorded during experiments are available for the prediction. This may be because the variation in measured dynamic responses, particularly lateral displacements, among the facilities was more or less influenced by a series of test procedures (e.g., how to build up the sloping ground) as well as the differences of input motions. However, the variation is hard to quantify in advance of predictions at the current moment, although the influence is non-negligible for the prediction accuracy. Thus, how to estimate the degree of variation in advance is left for future work as well as how to reduce experimental errors. To simulate the experimental results more realistically, type-C simulations may be required by identifying model parameters with trial and error for each facility's results. 


\section{References}

Alford, R. M., Kelly, K. R., \& Boore, D. M. (1974). Accuracy of finite difference modeling of the acoustic wave equation. Geophysics, 39(6), 834-842.

Arulanandan, K., \& Scott, R. F. (1993/1994). Verification of numerical procedures for the analysis of soil liquefaction problems. In Proceedings of the International Conference on the Verification of Numerical Procedures for the Analysis of Soil Liquefaction Problems (Vols. 1 and 2). Rotterdam: A. A. Balkema.

El Ghoraiby, M. A., Park, H., \& Manzari, M. T. (2017). LEAP 2017: Soil Characterization and 339 Element Tests for Ottawa F65 Sand. Washington, DC: The George Washington University,

El Ghoraiby, M. A., Park, H., \& Manzari, M. T. (2019). Physical and mechanical properties of Ottawa F65 Sand. In B. Kutter et al. (Eds.), Model tests and numerical simulations of liquefaction and lateral spreading: LEAP-UCD-2017. New York: Springer.

Hughes, T. J. R. (1980). Generalization of selective integration procedures to anisotropic and nonlinear media. International Journal for Numerical Methods in Engineering, 15, 1413-1418.

Iai, S. (2015). Liquefaction experiment and analysis projects (LEAP) through a generalized scaling relationship. In S. Iai (Ed). Geotechnics for catastrophic flooding events (pp. 95-97). London: CRC Press.

Iai, S., Matsunaga, Y., \& Kameoka, T. (1992). Strain space plasticity model for cyclic mobility. Soils and Foundations, 32(2), 1-15.

Iai, S., Morita, T., Kameoka, T., Matsunaga, Y., \& Abiko, K. (1995). Response of a dense sand deposit during 1993 Kushiro-Oki earthquake. Soils and Foundations, 35(1), 115-131.

Iai, S., Tobita, T., Ozutsumi, O., \& Ueda, K. (2011). Dilatancy of granular materials in a strain space multiple mechanism model. International Journal for Numerical and Analytical Methods in Geomechanics, 35(3), 360-392.

Iai, S., Ueda, K., Tobita, T., \& Ozutsumi, O. (2013). Finite strain formulation of a strain space multiple mechanism model for granular materials. International Journal for Numerical and Analytical Methods in Geomechanics, 37(9), 1189-1212.

Ishihara, K. (1996). Soil behaviour in earthquake geotechnics. New York, NY: Oxford University Press.

Ishihara, K., \& Yoshimine, M. (1992). Evaluation of settlements in sand deposits following liquefaction during earthquakes. Soils and Foundations, 32(1), 173-188.

Kutter, B. L., Carey, T. J., Hashimoto, T., Zeghal, M., Abdoun, T., Kokkali, P., Madabhushi, G., Haigh, S., d'Arezzo, F. B., Madabhushi, S., Hung, W. Y., Lee, C. J., Cheng, H. C., Iai, S., Tobita, T., Ashino, T., Ren, J., Zhou, Y. G., Chen, Y., Sun, Z. B., \& Manzari, M. T. (2018). LEAP-GWU-2015 experiment specifications, results, and comparisons. Soil Dynamics and Earthquake Engineering, 113, 616-628.

Kutter, B. L., Carey, T. J., Stone, N., Bonab, M. H., Manzari, M., Zeghal, M., Escoffier, S., Haigh, S., Madabhushi, G., Hung, W. Y., Kim, D.S., Kim N. R., Okamura, M., Tobita, T., Ueda, K., \& Zhou, Y. G. (2019a). LEAP-UCD-2017 V. 1.01 model specifications. In B. Kutter et al. (Eds.), Model tests and numerical simulations of liquefaction and lateral spreading: LEAP-UCD-2017. New York: Springer.

Kutter, B. L., Carey, T. J., Stone, N., Zheng, B. L., Gavras, A., Manzari, M., Zeghal, M., Abdoun, T., Korre, E., Escoffier, S., Haigh, S., Madabhushi, G., Madabhushi, S. S. C., Hung, W. Y., Liao, T. W., Kim, D. S., Kim, S. N., Ha, J. G., Kim, N.R., Okamura, M., Sjafuddin, A. N., Tobita, T., Ueda, K., Vargas, R., Zhou, Y. G., \& Liu, K. (2019b). LEAP-UCD-2017 comparison of centrifuge test results. In B. Kutter et al. (Eds.), Model tests and numerical simulations of liquefaction and lateral spreading: LEAP-UCD-2017. New York: Springer.

Kutter, B. L., Manzari, M. T., Zeghal, M., Zhou, Y. G., \& Armstrong, R. J. (2015). Proposed outline for LEAP verification and validation processes. In S. Iai (Ed). Geotechnics for catastrophic flooding events (pp. 99-108). London: CRC Press.

Lambe, T. W. (1973). Predictions in soil engineering. Geotechnique, 23(2), 151-201. 
Manzari, M. T., Kutter, B. L., Zeghal, M., Iai, S., Tobita, T., Madabhushi, S. P. G., Haigh, S. K., Mejia, L., Gutierrez, D. A., Armstrong, R. J., Sharp, M. K., Chen, Y. M., \& Zhou, Y. G. (2015). LEAP projects: Concept and challenges. In S. Iai (Ed). Geotechnics for catastrophic flooding events (pp. 109-116). London: CRC Press.

Ozutsumi, O., Sawada, S., Iai, S., Takeshima, Y., Sugiyama, W., \& Shimazu, T. (2002). Effective stress analyses of liquefaction-induced deformation in river dikes. Soil Dynamics and Earthquake Engineering, 22(9-12), 1075-1082.

Ueda, K. (2009). Finite Strain Formulation of a Strain Space Multiple Mechanism Model for Granular Materials and Its Application. Doctoral Thesis, Kyoto University, (in Japanese).

Ueda, K., \& Iai, S. (2018). Numerical predictions for centrifuge model tests of a liquefiable sloping ground using a strain space multiple mechanism model based on the finite strain theory. Soil Dynamics and Earthquake Engineering, 113, 771-792.

Ueda, K., Iai, S., \& Ozutsumi, O. (2015). Finite deformation analysis of dynamic behavior of embankment on liquefiable sand deposit considering pore water flow and migration. In Proceedings of the 6th International Conference on Earthquake Geotechnical Engineering, Christchurch, New Zealand. Paper No. 215.

Ueda, K., Iai, S., \& Tobita, T. (2011). Finite deformation analysis of earthquake induced damage to breakwater during 1995 Hyogoken-Nanbu Earthquake. In Proceedings of the 8th International Conference on Urban Earthquake Engineering, Tokyo, Japan (pp. 261-270).

Vargas, R. R., Tobita, T., Ueda, K., \& Yatsugi, H. (2019). LEAP-UCD-2017 Centrifuge Test at Kyoto University. In B. Kutter et al. (Eds.), Model tests and numerical simulations of liquefaction and lateral spreading: LEAP-UCD-2017. New York: Springer.

Zeghal, M., Manzari, M. T., Kutter, B. L., \& Abdoun, T. (2015). LEAP: Data, calibration and validation of soil liquefaction models. In Proceedings of the 6th International Conference on Earthquake Geotechnical Engineering, Christchurch, New Zealand. Paper No. 432.

Zienkiewicz, O. C., Taylor, R. L., \& Zhu, J. Z. (2000). The finite element method: Its basis and fundamentals (6th ed.). Amsterdam: Elsevier.

Open Access This chapter is licensed under the terms of the Creative Commons Attribution 4.0 International License (http://creativecommons.org/licenses/by/4.0/), which permits use, sharing, adaptation, distribution and reproduction in any medium or format, as long as you give appropriate credit to the original author(s) and the source, provide a link to the Creative Commons license and indicate if changes were made.

The images or other third party material in this chapter are included in the chapter's Creative Commons license, unless indicated otherwise in a credit line to the material. If material is not included in the chapter's Creative Commons license and your intended use is not permitted by statutory regulation or exceeds the permitted use, you will need to obtain permission directly from the copyright holder. 